\title{
Handoff Optimization for Wireless and Mobile Networks using Fuzzy Logic
}

\author{
Pooja Dhand \\ PhD Student \\ Maharishi Markandeshwar University \\ Mullana, Ambala
}

\author{
Parwinder Dhillon \\ PhD Student \\ Maharishi Markandeshwar University \\ Mullana, Ambala
}

\begin{abstract}
The most important issue in Personal Communication Services (PCS) is the mobility management. And the efficiency of this PCS system is dependent on the maintenance of a reliable and optimal radio link between the mobile user and the fixed system. When the mobile user moves out of his coverage area, handoff is required to enjoy continuation of services. In this paper, a handoff algorithm termed as Fuzzy controller for Handoff Optimization (FCHO) is introduced based upon fuzzy logic. Traditional algorithms for handoff using fixed values of parameters can perform well only in specific environment but FCHO exploits attractive features of several existing algorithms, and adds more capabilities to provide adaptation to the dynamic environment. Simulation results reveal that the proposed FCHO algorithm eliminates the problem of corner effect by dynamically changing the value of threshold and hysteresis with the change in the RSSI and the velocity of the mobile station.
\end{abstract}

\section{General Terms}

Wireless Networks and Mobile Networks

\section{Keywords}

Mobile station (MS), base station (BS), Fuzzy controlled handoff optimization (FCHO), Received signal strength indicator (RSSI).

\section{INTRODUCTION}

Handoff/Handover refers to the process of switching from one area of coverage or cell to another area of coverage or cell in case of weakening of a call in current state. It is a process of transferring the link from one channel to another either on same base station or another connected to same or different controller or switch.

The conventional handoff decisions are normally a single metric based i.e. RSSI, which alone is not suitable for current mobile system. In order to maintain a continuous and reliable link between the Mobile Station and the base station in a cellular network, new and better handoff algorithms are needed to keep QoS as high as possible. Handoff algorithms, based on soft computing techniques such as Fuzzy Logic, Neural Networks, genetic algorithms, probability theory etc can be used for the same purpose [1]. These kinds of algorithms are based on uncertainty, partial truth, and approximation and depend on the expertise advice for information gathering to reduce computational cost. The purpose of this work is to utilize fuzzy logic to model and solve a real world problem of handoffs. It also emphasizes on design and implement a generalized framework for the design of Fuzzy Logic based handoff controller.
The paper is organized as follows, Section 2 presents the related work in handoff; Section 3 presents the proposed handover optimization algorithm; Section 4 gives the results and performance analysis; and finally Section 5 concludes with future work.

\section{RELATED WORK}

No doubt with the increase in use of Personal Communication system, there is a race between new methods being proposed and discovered to remove problems associated with wireless communications like latency, bandwidth, reliability[2] etc. Many schemes have been proposed to reduce handoff latency in IEEE 802.11 wireless LANs.

$\operatorname{In}[3]$ authors have reduced handoff latency by reducing the number of APs to be scanned which is accomplished by cell sectoring and distance measurement with help of GPS but still cannot remove ping pong effect which can be may be minimized by using received signal strength method along with their proposed algorithm and hence left for future.

Much more work has been done to examine handover latency in Wireless Local Area Networks (WLAN) by which using signaling process for traffic control between $\mathrm{AP}$ and $\mathrm{MN}$ by authors in [4].

Work done by Ali Safa Sadiq, Kamalrulnizam Abu Bakar, and Kayhan Zrar Ghafoora explained in paper [5] includes two parameters: - a Received Signal Strength Indicator and the relative direction of a Mobile Node toward an access point, which are considered as inputs to the fuzzy logic system in order to facilitate the handover decision process and chose the best preferable access point around this MN. But traffic analysis and bandwidth has not been evaluated in their article hence left for future.

In [6] [7], more intelligent handover procedures, which consider other parameters than only the signal strength, have been considered. In [8], different handover policies for heterogeneous networks are used, considering as handover parameters mainly the air-interface type and the available bandwidth at the access router. In [9], fuzzy logic is considered to cope with the complexity of the information set retrieved from a heterogeneous mobile access network.

\section{PROPOSED FCHO ALGORITHM}

In this paper, a fuzzy logic-based hand-off initialization and decision making approach to achieve the optimal handover decision in wireless networks is proposed. Here, fuzzy logic is applied in order to select the most appropriate AP from the list of scanned APs that are available from the scanning phase, which is handled by the MS. Normally, the MS does not know which AP will be a good partner to perform a handover, and it can just depend on the quality of the signal strength, available 
link and other related information. In addition, sometimes, the MS will perform unneeded handovers during its roaming. Unnecessary handoff events results in following problems:-

1. Poor QoS

2. Wastage of channel bandwidth

3. Increase in system load

4. Causes call blocking and call termination

5. Packet loss

Therefore, in this paper, effort is taken to minimize unnecessary handoffs by taking into account appropriate handover initialization mechanism. Hence, FCHO algorithm is divided into two phases:

a. Handover initialization phase which is further divided into two subparts:-

i. To set the value of minimum threshold 'MT'.

ii. To set the value of hysteresis 'hys'.

This phase aims to remove the problem of corner effect. There can be many reasons for sudden decrease in the value of RSSI so this algorithm changes the value of threshold to facilitate or avoid handover as the case may be.

b. Handover decision making phase to decide the best and optimal APs from the scanned list of APs based on fuzzy logic.

The adaptive fuzzy system will aid the handover algorithm to obtain the best AP in the range, in terms of its signal strength, ensuring it is directed more towards the best AP; this is called AP-Optimal-Cost (AP-Cost).

\subsection{Phase I - Handoff Initialization Phase}

\subsubsection{Fuzzy controller for Threshold.}

The competence of FCHO lies in the fact that its value of threshold is constantly changing with the changing value of velocity of MS. The speed of the mobile station, its signal strength from the current $\mathrm{AP}$ and the last threshold are the inputs to the fuzzy controller. Feedback mechanism is used to better control the controller by knowing the probability of the previous value of threshold. 27 fuzzy rules have been designed to evaluate the value of MT.

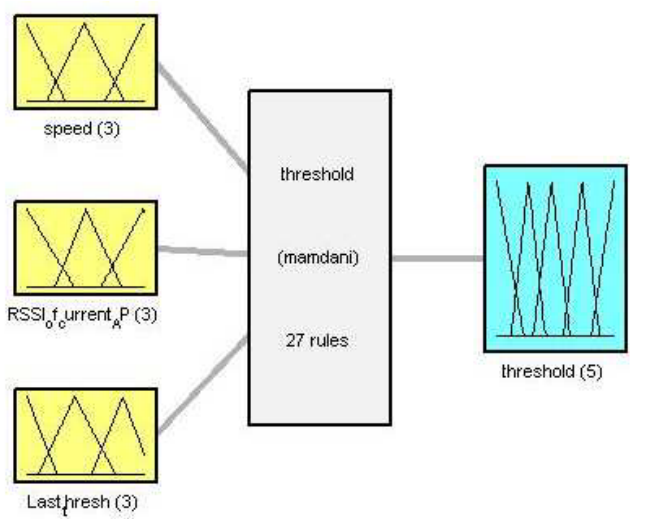

System threshold: 3 inputs, 1 outputs, 27 rules

Fig 1: FIS for Threshold
Following objectives are kept in mind while designing these rules [10]:

- If the signal strength is weak, the threshold will be increased to facilitate the handoff.

- If the signal strength is high, the threshold will be decreased to avoid unnecessary handoff.

- If the speed of the mobile station is slow and RSSI is high then threshold will be decreased to avoid unnecessary handoff.

- If the speed of the mobile station is fast and RSSI is weak then threshold will be increased to facilitate handoff.

- If the speed is fast but RSSI is strong then threshold will be decreased to avoid unnecessary handoff.

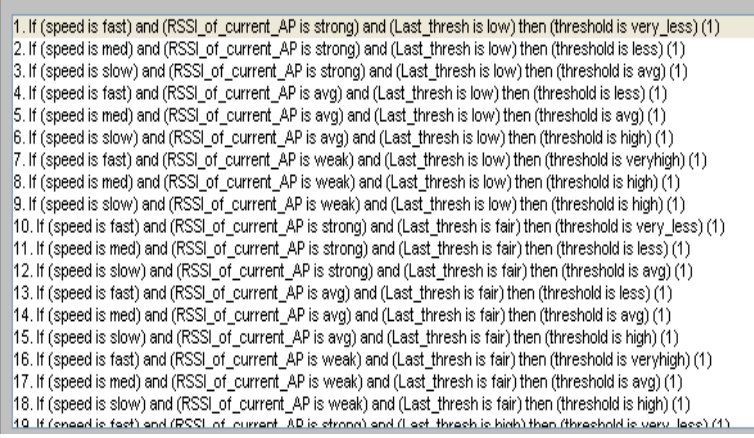

Fig 2: Fuzzy rules for evaluating minimum threshold 'MT'

The fuzzy inference engine consists of set of rules developed using expert knowledge and on member functions [11]. The above rules designed connect the inputs and the outputs based on a careful understanding of the real scenario behind the handover behaviors in wireless networks. In order to demonstrate the designed fuzzy inference system, one rule is used to show how the inference engine works and the outputs of each rule are combined to generate the fuzzy decision. If we consider a rule: "If RSSI is weak, speed is fast and last threshold is fair then the current minimum threshold is kept very high to facilitate handover" [12].

Defuzzification refers to the way a crisp value is extracted from a fuzzy set as a representation value. In our fuzzy decision making for 'MT', the centroid of area method for defuzzification is used. The defuzzification results will help the MS to select the accurate 'MT' value to handle the handover initialization phase.

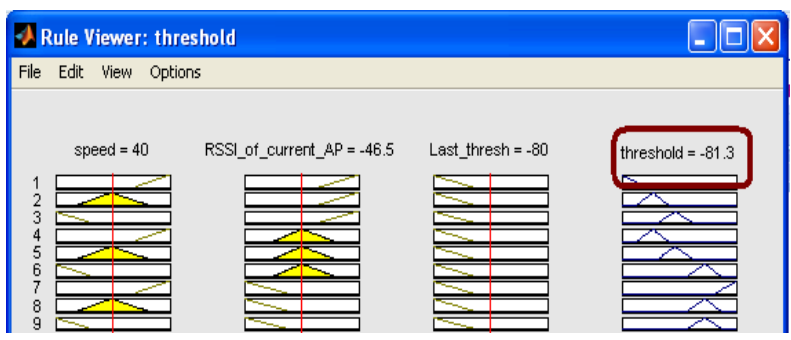

Fig 3: Output for minimum threshold 'MT'

\subsubsection{Fuzzy Controller for Hysteresis}

In this paper, a method for optimizing the value of hysteresis is proposed depending upon both the received signal strength and the traffic pattern. The signal strength of the transmitted signals of at least one base station (BS) of a neighboring cell 
is also measured and reported to the system. Hence, hysteresis can be defined as follows [10]:-

If RSSI [AP1] <RSSI [AP2] then

Handoff is required when RSSI [AP2]-RSSI [AP1] =hys

The two input variables to the fuzzy block are - the traffic pattern and the signal strength of the current AP and the output is hysteresis itself. For this case, the objectives for formulating the rules are:

- If the traffic pattern is high, it means that the acceleration of the mobile station is low; the hysteresis value is kept low with the purpose of facilitating the Handover.

- If the traffic pattern is medium, it means that the acceleration is moderate; the hysteresis value will be normal.

- If the traffic pattern is low, it means that the acceleration is high; the hysteresis value will be high to prevent unnecessary handoffs.

Nine rules are formulated for evaluating hysteresis as shown in Fig 4.

1. If (traffic_pattren is low) and (RSSI_of_neighbouring_AP is weak) then (hys is fair) (1) 2. If (traffic_pattren is low) and (RSSI_of_neighbouring_AP is mod) then (hys is fair) (1) 3. If (traffic_pattren is low) and (RSSI_of_neighbouring_AP is strong) then (hys is less) (1) 4. If (traffic_pattren is avg) and (RSSI_of_neighbouring_AP is weak) then (hys is fair) (1) 6. If (traffic_pattren is avg) and (RSSI_of_neighbouring_AP is strong) then (hys is less) (1) 7. If (traffic_pattren is high) and (RSSI_of_neighbouring_AP is weak) then (hys is high) (1) 8. If (traffic pattren is high) and (RSSI of neighbouring_AP is mod) then (hys is fair) (1) 9. If (traffic_pattren is high) and (RSSI_of_neighbouring_AP is strong) then (hys is less) (1)

Fig 4: Rules formulated for Hysteresis 'hys'

After Defuzzification the output is shown with the crisp value in Fig 5.

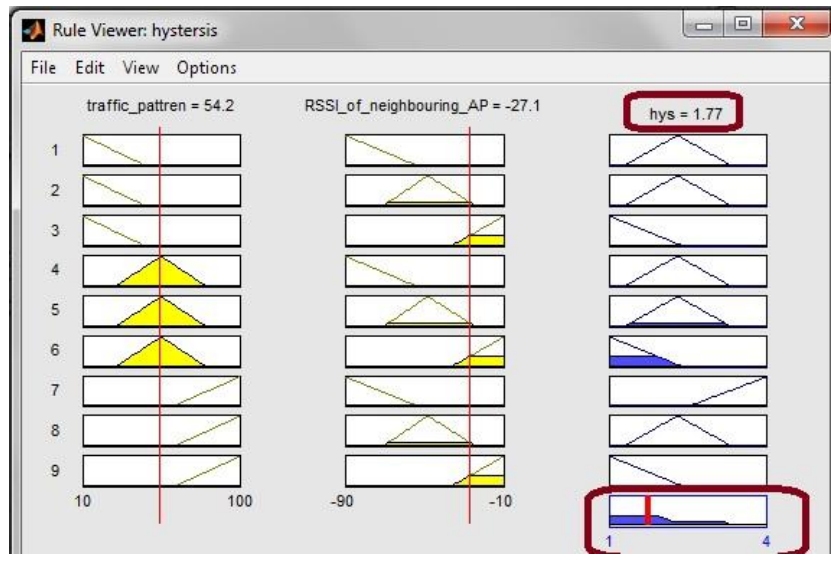

Fig 5: Output value of hysteresis when rules get fired

\subsection{Phase II- Handoff Decision making Phase}

\subsubsection{Optimal AP Handler}

When a mobile undergoes a handoff situation, it tries to look for another AP that guarantees a better RSSI. So, an Optimal AP handler is being designed which considers the RSSI of the APs, bandwidth availability and the physical distance between the mobile station and the AP as its input. This allows having a more efficient control of the handoff, discarding APs that do not fulfill the best expectations. The input variables are evaluated on the basis of 27 fuzzy rules which are decided after proper questionnaires and keeping in mind today's cellular environment. The output variable is referred to as AP_cost.

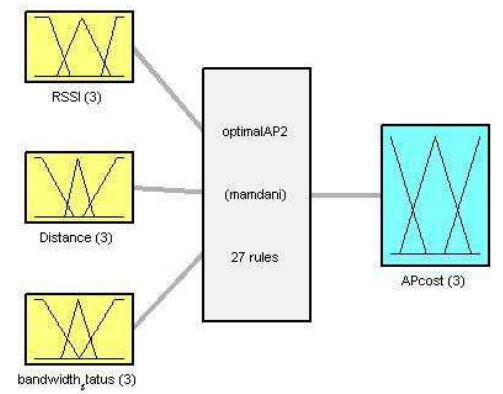

System optimal/AP2: 3 inputs, 1 outputs, 27 rules

Fig 6: FIS for AP_cost

Twenty seven rules are formulated for choosing best AP among the list of scanned APs as shown in Fig 6. These twenty seven rules form the most integral part of this work as if there is an error anywhere then it may cause the following problems:

1. Unnecessary handoff

2. Call blocking

3. Call termination

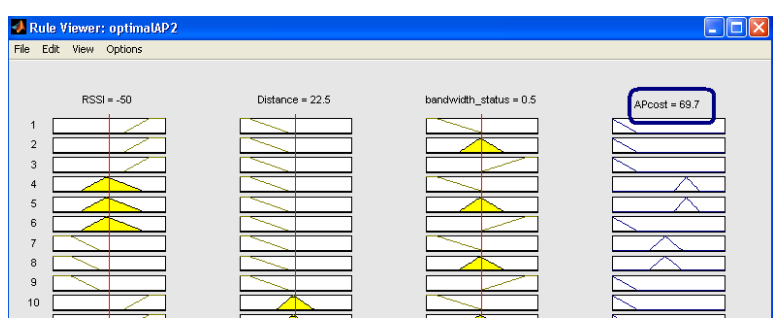

Fig 7: Rules for OptimalAP

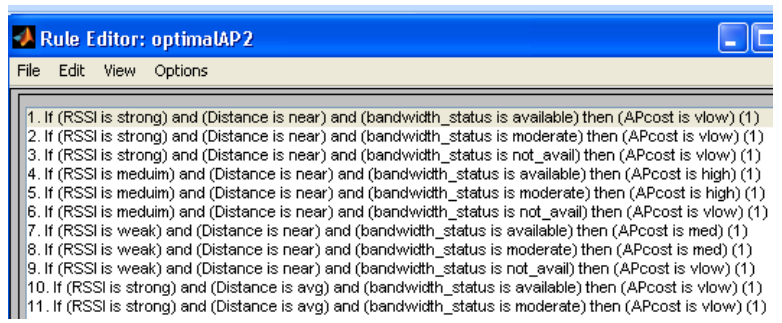

Fig 8: Output for evaluating OptimalAP

\section{RESULTS AND PERFORMANCE ANALYSIS}

The FCHO algorithm is designed to remove the ping pong problem, corner effect and to avoid the risk of unnecessary handovers by optimizing the values of thresholds and hysteresis. The M-file designed for this algorithm clearly depicts that the MS will enter into the Phase II only if the 
condition explained in section 3.1.2 is true otherwise a message appears that 'No handover required'. The relation between distance, bandwidth with $\mathrm{AP}$ _cost and relation between RSSI and distance with AP_cost is shown in Fig 1.9.
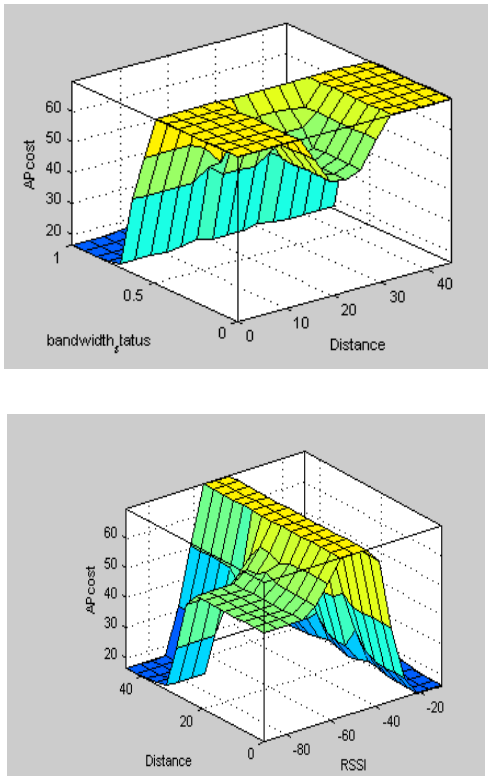

Fig 9 (a): Surface view showing relation between RSSI and distance with AP_cost. Fig 9(b): Surface view showing relation between distance and bandwidth with AP_cost.

\subsection{Comparison between Fixed and Adaptive RSS Algorithm}

In conventional handoff algorithms, fixed value of minimum threshold 'MT' and hysteresis 'Hys' is used. But in case of FCHO algorithm, dynamic values of 'MT' and 'Hys' according to the speed of mobile terminal and RSSI of the current AP and one of the neighbouring AP is calculated .The value of 'MT' is dependent on the speed of the mobile station and current RSSI value as shown in the following graph.

It is observed that with increase in velocity, 'MT' for adaptive handoff algorithm increases whereas 'MT' for fixed RSS algorithm remain constant Thus minimum threshold is decided on the basis of velocity of mobile terminal and it leads to low hand drop call rate as compared to fixed RSS.

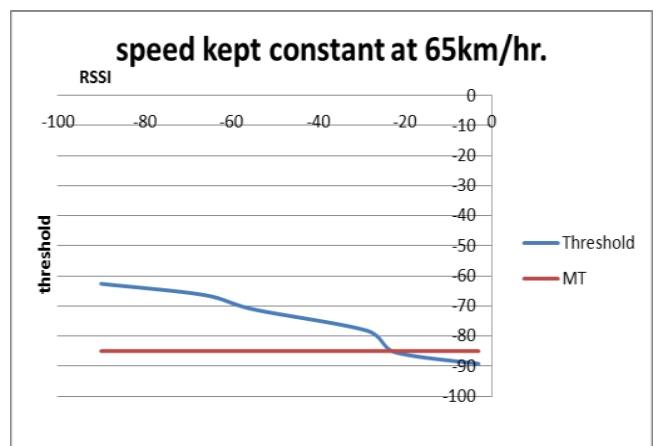

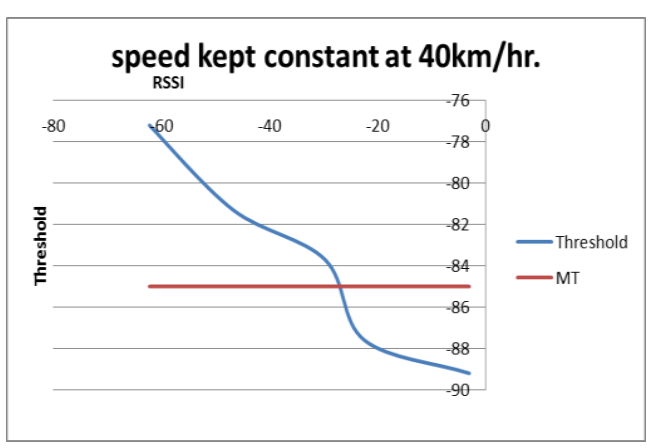

Fig 10 (a): Relation between RSSI and MT at average speed. Fig 10(b): Relation between RSSI and MT at high speed.
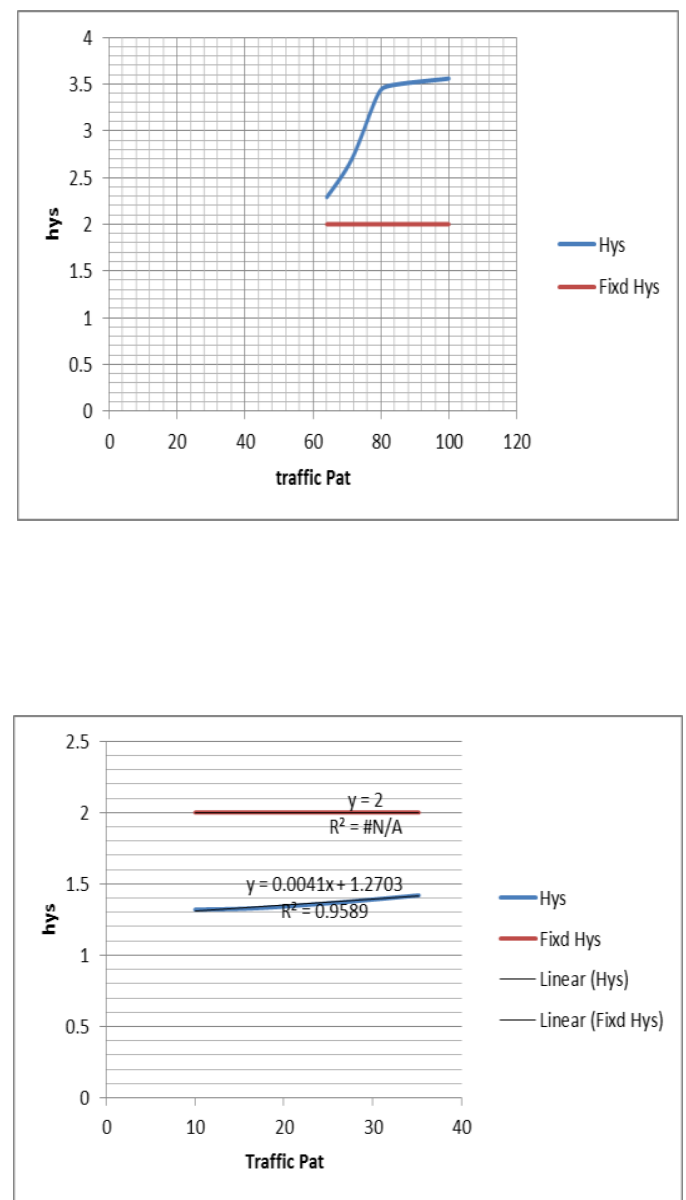

Fig 11(a): Relation between hys and traffic pattern at high RSSI. Fig 11(b): Relation between hys and traffic pattern at low RSSI.

Hence, this helps in reducing Ping-Pong effect and corner effect because as there is an abrupt decrease in the value of signal strength, the value of minimum threshold increases to facilitate handoff.

\section{CONCLUSION AND FUTURE WORK}

It's not the end but the beginning of a new research which will open many more dimensions and different visions for better handling mobility problems related to wireless and mobile computing. The proposed system can be reconfigured and additional features can be incorporated with increasing need of the customer and providing better quality of service, by 
adding additional parameters. For the future work, following modifications can be done to the proposed algorithm to improve the performance:-

1) Direction in which the MS is progressing can also be considered for improving the performance of the environment.

2) Corner effect and its problem must be elaborated and its remedy can be given.

3) FCHO algorithm can be extended to handle a heterogeneous environment.

\section{REFERENCES}

[1] A. A. Atayero, M. K. Luka. "Applications of Soft Computing in Mobile and Wireless Communications" International Journal of Computer Applications. Vol. 45, No. 22, PP. 54-56, 2012.

[2] H. Balakrishnan, S. Seshan, and R. H. Katz, "Improving Reliable Transport and Handoff Performance in Cellular Wireless Networks", ACM Wireless Networks Journal (WINET), Vol. 1, No. 4, PP. 469-481, 1995.

[3] D. Sarddar, S. Chatterje, U. Biswas, Mandovi Mukherjee, S. Mallik, P. Mazumder and M. K. Naskar. "Minimization of Handoff Latency by Cell Sectoring Method using GPS", International Journal of Computer Applications, Vol. 25, No. 4, PP. 22-29, 2011.

[4] N.S.V. Shet, K. Chandrasekaran and K.C. Shet, "Implementation of Handoff through wireless access point Techniques", Journal of Telecommunications, Vol. 2, No.. 2, 2010

[5] T. C. Ling, J. F. Lee, K. P. Hoh, "Reducing Handoff Delay In Wlan Using Selective Proactive Context Caching", Malaysian Journal of Computer Science, Vol. 23, No. 1, PP. 49-59, 2010.
[6] M. Stemm and R. Katz, "Vertical handoffs in wireless overlay networks", ACM Journal on Mobile Networks and Applications, Vol. 3, No. 4, PP. 335-350, 1998.

[7] K. Pahlavan et al, "Handoff in hybrid mobile data networks", IEEE Communication Magazine, Vol. 7, No. 2, PP. 34-46, 2000.

[8] H. J. Wang, R. H. Katz and J. Giese, "Policy- Enabled Handoffs across Heterogeneous Wireless Networks", In Proceedings of Second IEEE Workshop on Mobile Computing Systems and Applications (WMCSA '99), IEEE, PP. 51-60, 1999.

[9] P. Chan et al, "Mobility management incorporating fuzzy logic for a heterogeneous IP environment", IEEE Communication Magazine, Vol. 39, No. 12, PP. 42-51, 2001.

[10] D. Pubill, A. I. Perez, "Handover Optimization with Fuzzy Logic in 802.11 Networks", In Proceedings of conference on Information Processing and Management of Uncertainty in Knowledge-Based Systems (IPMU), Paris (France) , 2006.

[11] C. F. Kwong, T. C. Chuah and S. W. Lee, "Adaptive Network Fuzzy Inference System (ANFIS) Handoff Algorithm", In Proceedings of International conference on Future Computer and Communication (ICFCC), Malaysia, PP. 195-198, 2009.

[12] A. S. Sadiq, K. A. Bakar, K. Z. Ghafoor, "A Fuzzy Logic Approach for Reducing Handover Latency in Wireless Networks", Network Protocols and Algorithms, Vol 2, No. 4, PP. 61-87, 2010. 